\title{
界面扩张流变方法研究大分子与表面活性剂的相互作用
}

\author{
朱艳艳＼cjkstart徐桂英” \\ (山东大学胶体与界面化学教育部重点实验室, 济南 250100)
}

摘要: 结合本课题组的工作, 较系统地总结了近年来有关界面扩张流变技术在大分子/表面活性剂混合体系研 究中的应用. 大量研究表明, 通过分析界面扩张粘弹性数据和界面弛豫过程的特征参数可以研究界面层微观性 质, 从而阐明大分子/表面活性剂的相互作用机理, 这对实际生产和应用具有重要的指导意义.

关键词：大分子；表面活性剂；界面扩张流变；相互作用

中图分类号： O648; O647

\section{Investigation of Macromolecule/Surfactant Interaction by Interfacial Dilational Rheology Method}

\author{
ZHU Yan-Yan XU Gui-Ying * \\ (Key Laboratory of Colloid and Interface Chemistry, Ministry of Education, Shandong University, Jinan 250100, P. R. China)
}

\begin{abstract}
The applications of interfacial dilational rheology method in studying macromolecule/surfactant system were systematically summarized combing with our work. Many previous studies have indicated that this method can be used to probe the microcosmic property of interfacial layer. This is done by analyzing the interfacial dilational viscoelasticity and characteristic parameters of the interfacial relaxation process. The interaction mechanism between macromolecule and surfactant could thus be investigated. The method can be applied in practice.
\end{abstract}

Key Words : Macromolecule; Surfactant; Interfacial dilational rheology; Interaction

在食品、医药、化妆品生产以及采矿和油田开发 过程中常常将表面活性剂与大分子物质复配应用, 使用这种复配体系可以减少大分子或者表面活性剂 的用量, 显著提高体系的功效. 并且由于二者之间存 在疏水或者静电相互作用还可能使体系具有许多独 特的性质, 例如体系的粘度、界面吸附、增溶和药物 传输等性能都不同于单一组分体系, 因此表面活性 剂/大分子混合体系的研究一直是人们非常感兴趣 的研究课题 ${ }^{[1-4]}$. 研究表面活性剂与大分子相互作用 的方法很多 ${ }^{[4-13]}$, 通过不同技术考察表面活性剂/大 分子混合体系的界面特性研究两者的相互作用是最 常用的方法 ${ }^{[4,14]}$. 表面张力法是研究两者混合体系界 面行为的最经典、方便和有效的方法. 它可以得到表
面活性剂在界面的吸附量、极限分子占据面积以及 两者相互作用的临界浓度和结合量等信息 ${ }^{[15,16]}$. 但表 面张力方法通常更适合研究相互作用较弱的体系 ${ }^{[4]}$, 而且, 表面活性剂/大分子混合体系的界面特性既取 决于两者的竞争(或协同)吸附,也依赖于所形成界 面膜的结构、形态和动力性质 ${ }^{[17]}$. 界面流变技术是近 年来发展起来的研究界面层动态性质的重要工具, 它可以提供溶液界面上可溶性吸附膜和不溶膜粘弹 性质的信息 ${ }^{[4]}$. 与体相流变学相似, 界面流变学主要 涉及到应力、形变和形变速率的关系. 根据形变方式 的不同, 界面流变学主要可分为两种: 界面扩张流 变 ${ }^{[18]}$ 和界面剪切流变 ${ }^{[19]}$. 界面扩张流变对表面活性 物质的吸附和脱附动力学更为敏感 ${ }^{[20]}$, 它包括大分

Received: September 8, 2008; Revised: October 9, 2008; Published on Web: December 4, 2008.

*Corresponding author. Email: xuguiying@sdu.edu.cn; Tel: +86531-88365436.

山东省自然科学基金(Y2007B32)资助项目

C Editorial office of Acta Physico-Chimica Sinica 
子在界面上的构型变化和链段重组等; 界面剪切流 变则对吸附层内分子间相互作用和界面上网络结构 形成更为敏感 ${ }^{[17]}$. 因此通过界面流变性测定可研究 界面层中表面活性剂与大分子的相互作用 ${ }^{[4]}$. 与界 面剪切粘弹性质相比, 无论是实验还是理论的研究 都表明, 扩张性质比剪切性质要大几个数量级 ${ }^{[2,122]}$.

界面膜的扩张粘弹性质以及界面物质的交换在 许多工业领域中都至关重要, 如: 含有明胶和表面 活性剂的感光乳剂的涂装过程和矿物浮选工艺等. 界面粘弹性也对泡沫、乳状液的形成和稳定起着重 要作用, 例如三次采油乳状液液滴间界面膜强度的 大小是决定乳状液稳定性的关键 ${ }^{[23]}$. 界面扩张粘弹 性反映了界面膜抵抗扩张形变的能力, 它产生的微 观基础是发生在界面及其附近的微观弛豫过程, 研 究界面扩张粘弹性对阐明大分子/表面活性剂的相 互作用机理具有重要意义. 本文结合本课题组的工 作, 着重讨论了界面扩张流变法在大分子/表面活性 剂体系的近期研究进展, 以期为表面活性剂/大分子 混合体系在食品、日用化工以及油田开发等领域的 合理应用提供基础数据.

\section{1 实验方法}

常见的界面扩张粘弹性测量方法有振荡气泡/ 悬滴法和 Langmuir 槽法 ${ }^{[24-31]}$. 不同方法的频率适用 范围不同. 其中, 振荡气泡法由 Lunkenhemier 发明, 它测量的是当通过振荡改变气相体积时从而导致气 相中气体的压力变化, 后来柏林/波茨坦的 MPRI 和 Florenze 大学进一步发展了该方法 ${ }^{[32]}$. Langmiur 槽 法是通过滑障运动来进行界面的压缩或扩张, 同时 用 Wilhelmy 吊片直接测得界面张力的变化.

\section{2 理论基础}

界面扩张流变考察界面形变大小以及形变速率 与张力之间的二维函数关系, 当界面受到小幅谐波 扰动时, 界面扩张模量 $(E)$ 定义为表面压 $(\pi)$ 与面积 变化 $(\Delta A)$ 之间的比值 ${ }^{[33]}$ :

$$
E=\frac{\mathrm{d} \pi}{\mathrm{d} \ln A} \approx-A \frac{\Delta \pi}{\Delta A}
$$

界面扩张模量是一个与界面扩张频率 $(\omega)$ 有关的复 数量, 即:

$$
\begin{gathered}
E=E \exp (\mathrm{i} \omega t)=E_{\mathrm{r}}+\mathrm{i} E_{\mathrm{i}}=E_{\mathrm{d}}+\mathrm{i} \omega \eta_{\mathrm{d}} \\
\tan \theta=\frac{\omega \eta_{\mathrm{d}}}{E_{\mathrm{d}}}
\end{gathered}
$$

式中, $E_{\mathrm{d}}$ 是界面扩张弹性模量, 或称储能模量; $\eta_{\mathrm{d}}$ 是
界面扩张粘度, $\omega \eta_{\mathrm{d}}$ 是粘性模量, 或称损耗模量; $\theta$ 是 相角.

相角是界面膜粘弹特性的定量表征, 反映了粘 性部分和弹性部分的比值. 相角越小, 表示界面膜弹 性越强. 其中储能模量, 其来源是界面分子因扰动偏 离平衡状态导致的能量改变, 与分子间相互作用密 切相关; 损耗模量与表面活性剂分子在界面与体相 间的交换、界面分子排布方式的改变等弛豫过程相 关 ${ }^{[34]}$. 扩张实验的工作频率是影响扩张粘弹参数的 重要因素. 关于扩张频率的影响有两种极限情况, 很 低的扩张频率使得单层膜有足够的时间恢复平衡, 当扩张频率为 0 时, 界面张力的变化为零, 因此界面 扩张模量等于零; 当扩张频率较大时, 给予被扰动 的界面吸附膜通过扩散交换等驰豫过程重新恢复平 衡的时间就越短, $E$ 值就较大. 同时, 由于弛豫过程 作用时间较短, 贡献较小, 粘性部分所占比例就较 低, 相角逐渐降低. 无穷大的扩张频率使得表面活性 剂分子在体相与界面之间的扩散交换可以忽略, 界 面膜类似于不溶性单层膜, 这种膜是完全弹性膜, 其 $E$ 值等于Gibbs弹性 $\left(E_{\mathrm{G}}\right)$. 因此, $E$ 值随着频率的逐渐 增大而增加, 直至达到平台值 ${ }^{[35]}$.

对于低分子量表面活性剂, 通常认为弛豫过程 主要是分子在体相与界面的扩散交换, 且弛豫时间 通常比较短. 大分子的界面粘弹行为显著不同于传 统的低分子表面活性剂. 虽然大分子从体相到表面 层的扩散交换过程可以忽略, 但是必须考虑单体分 子在表面层的不同位置之间的扩散交换过程 ${ }^{[3]}$. Noskov 等 ${ }^{[37-39}$ 认为在表面膜内存在两个微区: 一个 是紧贴着气相、比较窄的浓度区域, 称为最接近微区 (the proximal region); 另一个是单体分子的总浓度 很低, 形成嵌人到液相的尾状(tail)和环状(loop)结构 的区域, 称为末端微区(the distal region). 表面张力的 大小主要决定于 “最接近微区”中单体浓度的大小. 大分子链内形变产生的慢弛豫过程和分子链段的扩 散交换引起的快弛豫过程是大分子界面现象的基 础. 据此可以用来解释大分子扩张模量随其浓度的 变化.

\section{3 界面扩张流变法研究不同大分子与表面 活性剂的相互作用}

\section{1 生物大分子/表面活性剂混合体系}

\subsection{1 蛋白质/表面活性剂混合体系}

界面流变技术最早主要是应用于食品工业中蛋 
白质吸附层对分散体系稳定性方面的研究 ${ }^{[40]}$. 在实际 体系中使用蛋白质/表面活性剂混合体系非常普遍, 由于蛋白质与表面活性剂的相互作用能显著地改变 界面吸附层的性质, 因此, 近年来人们对有关界面流 变学方法研究二者相互作用的报道倍感兴趣, 它可 以提供有关分子在气/液和油/水界面的竞争吸附、蛋 白质构象变化和界面膜紧密程度及强度的信息 ${ }^{[41]}$.

单独的蛋白质分子在界面上的吸附呈现不同的 状态: 表面压较小时, 分子占据较大的摩尔表面面 积; 表面压较大时, 分子则占据较小的摩尔表面面 积. 这意味着随着蛋白质浓度的增加, 表面层厚度越 来越大 ${ }^{[42]}$. 但当体系中同时含有非离子表面活性剂 时, 由于蛋白质和表面活性剂之间主要是疏水相互 作用(有时也存在氢键相互作用), 所形成复合物的表 面活性比较差, 甚至低于单纯蛋白质的活性, 因此吸 附层主要是蛋白质和表面活性剂二者之间的竞争吸 附. 而从特定的表面活性剂浓度开始, 界面上几乎全 部为非离子表面活性剂 ${ }^{[42]}$. 由于复合物构象的变化 和界面上蛋白质的解吸作用, 界面粘弹性参数随着 蛋白质和表面活性剂的摩尔比而改变 ${ }^{[43]}$.

Patino 等 ${ }^{[44]}$ 用 Langmuir 槽法研究了 $\beta$-乳球蛋 白和甘油一酸酯混合吸附膜和伸展膜的界面扩张流 变行为, 发现界面扩张性质依赖于界面组成和表面 压 $(\pi)$. $\beta$-乳球蛋白吸附膜的 $E$ 值随着 $\pi$ 的增大而增 加, 直到 $\pi$ 大于蛋白质的崩溃压 $\pi_{\mathrm{e}}, E$ 值保持不变, 这种增加是由单层分子之间相互作用的增强引起 的. 随着蛋白质体相浓度的增加 $\left(1 \times 10^{-7}-1 \times 10^{-6}(\mathrm{w}\right.$, 质 量分数)), 蛋白质吸附膜的 $E$ 值逐渐增大; 而且伸展 膜的 $E$ 值大于吸附膜的. 其原因可能是 $\beta$-乳球蛋白 单层伸展过程中蛋白质的伸展导致三级结构损失, 具有环状结构的氨基酸残基相互作用增强所致. $\pi$ 小于 $\pi_{\mathrm{e}}$ 时, 蛋白质伸展膜的相角小于吸附膜, 这表 明蛋白质伸展有利于具有高弹性特征的界面凝胶结 构的形成; 而 $\beta$-乳球蛋白 $\left(1 \times 10^{-5}(w)\right)$ 和甘油棕㭣酸 酯混合体系吸附膜和伸展膜的 $E$ 值大于单纯 $\beta$-乳 球蛋白吸附膜数值, 并远小于甘油棕㭣酸酯伸展膜 数值, 这说明 $\beta$-乳球蛋白和甘油棕㭣酸酯在吸附膜 和伸展膜中是共存的. 当 $\pi$ 大于 $\pi_{\mathrm{e}}$ 时, 伸展膜的相 角又增加, 表明多层结构的形成; 而混合体系的 $E$ 值低于单纯甘油棕㭣酸酯, 说明单层中仍有少量 $\beta$ 乳球蛋白. 布鲁斯特角 $(\mathrm{BAM})$ 也证实了这种结果. 当 用甘油油酸脂代替甘油棕㭣酸酯时, 混合体系的吸 附膜 $E$ 值却几乎等于单纯 $\beta$-乳球蛋白吸附膜之值, $\pi$ 较高时混合层的 $E$ 值仍由蛋白质决定, 混合体系 单层呈现粘弹性. 这说明甘油棕榈酸酯顶替界面上 蛋白质分子的能力大于甘油油酸脂.

Blomqvist 等 ${ }^{[45]}$ 研究了 $1 \times 10^{-5} \mathrm{~mol} \cdot \mathrm{L}^{-1} \beta$-乳球蛋 白和 F127( $\left.\mathrm{PEO}_{99} \mathrm{PPO}_{65} \mathrm{PEO}_{99}\right)$ 混合吸附膜扩张模量 $(\omega=0.13 \mathrm{~Hz})$ 随着时间和 $\pi$ 的变化. 单纯的蛋白质吸 附膜的 $E$ 值随着时间的延长而逐渐增大, 在 $1 \mathrm{~h}$ 时 达到 $130 \mathrm{mN} \cdot \mathrm{m}^{-1}$, 表明界面上形成了机械强度很高 的分子间网络结构. $E$ 值随着 $\pi$ 的增大而不断增加, 这是因为蛋白质分子的伸展使得更多的疏水部分暴 露在空气中和分子间网络结构的形成. 随着 F127 浓度增大, 同等时间和 $\pi$ 时的 $E$ 值逐渐减小, 界面 流变行为逐渐由类似蛋白质向类似共聚物行为转 变. 混合体系的吸附膜均以弹性为主. 当 F127 的浓 度为 $2 \times 10^{-8} \mathrm{~mol} \cdot \mathrm{L}^{-1}$ 时, 尽管此时表面主要是蛋白 质, 但是 F127 已经对界面粘弹性产生了影响. 当 F127 的浓度为 $2 \times 10^{-7}$ 和 $5 \times 10^{-7} \mathrm{~mol} \cdot \mathrm{L}^{-1}$ 时, 随着时 间的延长首先形成具有粘弹性的蛋白质层, 之后 F127 不断吸附到界面上, $E$ 值经过最大值, 然后随 时间的延长又减少, 说明 F127 替代了界面上的蛋 白质分子. 当 $\mathrm{F} 127$ 的浓度为 $2 \times 10^{-6} \mathrm{~mol} \cdot \mathrm{L}^{-1}$ 时, $\beta$-乳 球蛋白和 F127 共同吸附形成混合界面层, 蛋白质 分子还未被完全替代, 这一点从表面张力数据不易 看出.

近年来 Miller 等 ${ }^{[46]}$ 对表面活性剂/蛋白质混合 体系的理论研究方面做出了突出的贡献. 他们通过 对 Jiang 和 Joos 模型的偏导数部分进行小的修正, 并考虑了表面活性剂分子在界面上的倾斜和分子可 压缩性等因素对 Lucassen 和 van del Tempel(单组分 体系)进行修正之后, 发现这些不同方法的结果是等 同的, 并且对研究混合体系界面膜行为都是很有效 的. 应用以上模型, 他们还对十烷基二甲基氧化膦 $\left(\mathrm{C}_{10} \mathrm{DMPO}\right)$ 和 $\beta$-乳球蛋白混合体系的界面流变行 为的计算和实验结果进行了比较: 计算结果表明, 当 $\mathrm{C}_{10} \mathrm{DMPO}$ 浓度较低时, 被 $\beta$-乳球蛋白覆盖的表 面面积所占比例高达 $98 \%$, 当 $\mathrm{C}_{10} \mathrm{DMPO}$ 浓度为 $2 \times$ $10^{-3} \mathrm{~mol} \cdot \mathrm{L}^{-1}$ 时, 被二者覆盖的表面面积所占比例几 乎相等; 而实验结果表明, 随着体相中 $\mathrm{C}_{10} \mathrm{DMPO}$ 浓 度从 $2 \times 10^{-5} \mathrm{~mol} \cdot \mathrm{L}^{-1}$ 增加到 $7 \times 10^{-5} \mathrm{~mol} \cdot \mathrm{L}^{-1}$, 混合体 系的 $E$ 值逐渐减少, 而且相角随着 $\mathrm{C}_{10} \mathrm{DMPO}$ 浓度 的增加呈现先减小后增加的趋势, 与理论计算结果 一致.

蛋白质与离子表面活性剂通过静电相互作用所 
形成的复合物通常具有比单纯蛋白质和表面活性剂 分子较高的表面活性, 仅加人少量的离子表面活性 剂就可以明显改变吸附层蛋白质的结构. 随着体相 中表面活性剂的进一步增大, 更多的表面活性剂通 过疏水相互作用与蛋白质/表面活性剂复合物结合, 从而使得形成的复合物亲水性较强, 表面活性降低. 由于在表面上存在竞争吸附, 越来越多的复合物被 表面活性剂分子所取代 ${ }^{[2]]}$.

Rao 等 ${ }^{[47]}$ 用振荡气泡法研究了十二烷基硫酸钠 (SDS) 和高浓度 $(7 \%(w))$ 明胶的界面扩张流变行为. 图 1 显示了明胶/SDS 混合体系的界面流变参数变 化. 随着 SDS 浓度的不断增加, 吸附膜的 $E$ 值逐渐 减小, 当 $\operatorname{SDS}$ 体相浓度大于 $1 \times 10^{-3} \mathrm{~mol} \cdot \mathrm{L}^{-1}$ 后, 吸附 膜的性质几乎与单纯 SDS 相同. 当 SDS 浓度低于 $1 \times 10^{-3} \mathrm{~mol} \cdot \mathrm{L}^{-1}$ 时, 所测的相角几乎不变, 这就表明 吸附膜本质上是弹性的. 作者认为 SDS 的加人导致 明胶吸附层扩张弹性值减小是由于明胶在界面上的 网络结构被破坏.网络结构破坏的原因有三: (i) SDS 分子荷负电的头基与明胶链上的精氨酸和赖氨酸残 基通过静电作用结合，而且不仅 SDS 本身分子之间 有疏水作用, SDS 也会和明胶链上的亮氨酸、异亮 氨酸和蛋氨酸疏水残基相结合. SDS 结合到明胶链 上带正电荷的残基就会中和明胶链上的电荷, 从而 屏蔽了离子化基团的静电作用; (ii) SDS 结合到氨 基残基上之后可形成氢键的作用点减少; (iii) SDS

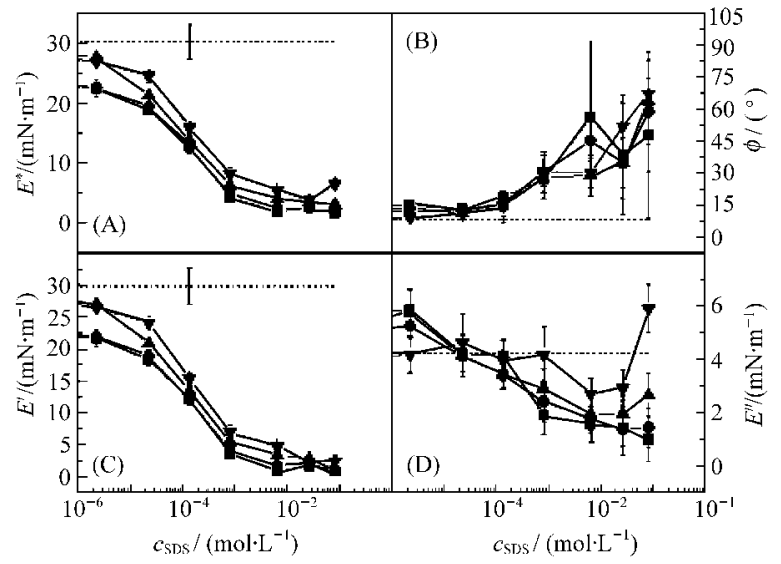

图 $140{ }^{\circ} \mathrm{C}$ 不同频率时明胶/SDS 混合体系的界面流变 参数变化 ${ }^{[47]}$

Fig.1 Interfacial dilational viscoelastic parameters of gelatin/SDS mixed system at different frequencies at $40{ }^{\circ} \mathrm{C}^{[47]}$

(A) complex surface moduli $E^{*}$, (B) phase angle $\phi$, (C) storage

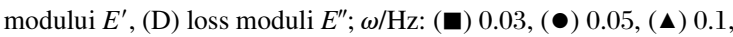

( $\mathbf{v})$ 0.25; SDS: sodium dodecyl sulfate
分布在吸附层内造成自然破坏, 这一结果已被中子 反射实验所证实 ${ }^{[48]}$. 这些效应在宏观上即表现为吸 附层的 $E$ 值下降.

我们研究了明胶与不同阳离子表面活性剂混合 体系在气/液界面上的扩张粘弹性 ${ }^{[49]}$. 发现单纯烷基 三甲基溴化铵型双子表面活性剂 $\left(\mathrm{C}_{12} \mathrm{C}_{2} \mathrm{C}_{12}\right)$ 浓度达到 $2.5 \times 10^{-6} \mathrm{~mol} \cdot \mathrm{L}^{-1}$ 时, 膜弹性和强度达到最大; 而明 胶浓度低于 $0.1 \mathrm{~g} \cdot \mathrm{L}^{-1}$ 时, 吸附在界面上的明胶分子 近乎以“平躺”的二维构象形式存在, 不能形成长的 尾状或环状结构, 随明胶浓度增加, 界面上的明胶分 子数目逐渐增加, 但其界面浓度的增加阻碍了部分 尾状或环状结构的明胶分子的完全伸展, 故明胶的 $E$ 值随浓度增大而增大, 并在 $1 \mathrm{~g} \cdot \mathrm{L}^{-1}$ 处出现一个极 大值. 明胶浓度大于 $1 \mathrm{~g} \cdot \mathrm{L}^{-1}$ 时, 由于 “最接近区域” 和“末端区域”之间的扩散交换加快, 使得明胶的 $E$ 值大大降低. 阳离子表面活性剂的加入能显著地改 变明胶的界面粘弹性质. 当 $0.05 \mathrm{~g} \cdot \mathrm{L}^{-1}$ 明胶存在时, 体系的扩张模量和扩张弹性达到最大值所对应的 $\mathrm{C}_{12} \mathrm{C}_{2} \mathrm{C}_{12}$ 浓度为 $1.0 \times 10^{-5} \mathrm{~mol} \cdot \mathrm{L}^{-1}$. 而且, 当 $\mathrm{C}_{12} \mathrm{C}_{2} \mathrm{C}_{12}$ 浓度小于 $2.5 \times 10^{-4} \mathrm{~mol} \cdot \mathrm{L}^{-1}$ 时, 体系的相角对浓度的 依赖性较小, 高于此浓度时, $\mathrm{C}_{12} \mathrm{C}_{2} \mathrm{C}_{12}$ 明胶体系的相 角随浓度的变化较大. 当 $\mathrm{C}_{12} \mathrm{C}_{2} \mathrm{C}_{12}$ 的浓度超过其 $\mathrm{cmc}$ 时, 体系的 $E$ 值、相角与相同浓度的表面活性 剂溶液的 $E$ 值、相角的数值比较接近, 说明最终界 面膜上的明胶分子完全被表面活性剂分子 $\mathrm{C}_{12} \mathrm{C}_{2} \mathrm{C}_{12}$ 所取代. 这表明随 $\mathrm{C}_{12} \mathrm{C}_{2} \mathrm{C}_{12}$ 浓度增加,明胶和 $\mathrm{C}_{12} \mathrm{C}_{2} \mathrm{C}_{12}$ 之间的静电和疏水相互作用将界面上的明胶分子逐 渐“拉人”体相中, 界面上最终被 $\mathrm{C}_{12} \mathrm{C}_{2} \mathrm{C}_{12}$ 分子所占 据. 对比十二烷基三甲基溴化铵(DTAB)/明胶和十六 烷基三甲基溴化铵(CTAB)/明胶体系的界面粘弹性 结果发现, 尽管随着表面活性剂浓度增加, 混合体系 的 $E$ 值均出现一个最大值, 但出现最大值所对应的 表面活性剂浓度不同, 扩张模量最大值的数值也不 同, 其大小顺序依次为明胶 $/ \mathrm{C}_{12} \mathrm{C}_{2} \mathrm{C}_{12}$ 体系 $>$ 明胶/ $\mathrm{CTAB}>$ 明胶/DTAB 体系.

Latnikova 等 ${ }^{[50]}$ 研究了 F68 $\left(\mathrm{PEO}_{76} \mathrm{PPO}_{29} \mathrm{PEO}_{76}\right)$ 、 DTAB 和 SDS 对 $\beta$-酪蛋白动态界面粘弹性的影响. 结果表明, 单纯 $\beta$-酪蛋白的动态界面粘弹性随时间 的变化出现两个最大值, 这对应于蛋白质亲水和疏 水两部分从“最接近区域”到以环状或者尾状形式存 在的“末端区域”结构的连续转变. 第一个最大值出 现区域是蛋白质的亲水部分形成中间或者末端部 分, 以环状形式进人亚相, 蛋白质片段在“最接近区 
域”和“末端区域”之间发生扩散交换, 界面扩张模量 值下降; 第二个出现区域是蛋白质的疏水部分以环 状或者尾状形式进人亚相. 离子表面活性剂对 $\beta$-酪 蛋白动态界面流变性质的影响是由表面活性剂与蛋 白质的带相反电荷的基团之间的相互作用引起的. 随着 DTAB 浓度的增加, 扩张模量的第一个最大值 先增加后减小; 而第二个最大值随着 DTAB 的加人 不断减小直至消失. 随 SDS 浓度的增加, 扩张模量 的第一个最大值不断减小直至消失, 而第二个最大 值则不断增大, 当 $\mathrm{SDS}$ 的浓度大于 $1.6 \times 10^{-4} \mathrm{~mol} \cdot \mathrm{L}^{-1}$ 时, 仅可看到第二个最大值, 而且随着 SDS 的加人 不断减小. 当 $\mathrm{pH}=7$ 时, $\beta$-酪蛋白带负电荷, 这部分 电荷主要属于 $\mathrm{N}$-终端的亲水部分, $\beta$-酪蛋白/DTAB 复合物疏水性增强, 阻止了环状或者尾状结构进人 临近区域, 第一个最大值增大; 随 DTAB 浓度增加, 蛋白质亲水和疏水两部分的差别越来越小, 第二个 最大值消失, 而蛋白质/DTAB 复合物亲水性增强, DTAB 在界面上的吸附量增加, 第一个最大值不断 减小. SDS 的加人使得整个蛋白质/SDS 的复合物疏 水性增强, 因此亲水部分对动态界面流变性质的影 响减弱, 直至第一个最大值消失. 而 F68 与 $\beta$-酪蛋 白的作用机理是前者逐渐取代吸附层内的蛋白质, 使得界面流变行为逐渐由蛋白质控制转向由共聚物 控制.

\subsection{2 多糖类高分子/表面活性剂混合体系}

多糖类生物高分子主要包括黄原胶、壳聚糖、纤 维素和淀粉等几种类型. 与传统化学工业中的合成 高分子相比, 多糖类生物高分子具有许多诱人的特 性和奇妙的功能 ${ }^{[11-53]}$. 它们来源于天然产物, 不仅在 工业上应用可以实现可持续性, 而且对环境友好.

Babak 等 ${ }^{[54]}$ 通过振荡气泡法对比研究了壳聚糖 $(\mathrm{Ch}) 、$ 疏水修饰的壳聚糖 $\left(\mathrm{C}_{12} \mathrm{Ch}\right)$ 和壳聚糖与 SDS 混 合体系(Ch-SDS)的界面扩张粘弹性在相同频率下随 时间的变化. 结果表明, $\mathrm{Ch}$ 的界面扩张弹性最大数 值几乎为 0 , 而 $\mathrm{C}_{12} \mathrm{Ch}$ 和 Ch-SDS 分别为 60 和 80 $\mathrm{mN} \cdot \mathrm{m}^{-1}$. 导致大分子吸附层界面扩张弹性差异的主 要原因可能有以下两方面: (i) 大分子相互作用导致 吸附能量增加; (ii) 大分子横向相互作用 (内聚力). 在测定时间内弹性模量随着 $\pi$ 的增大而增加, $\mathrm{Ch}$ 和 $\mathrm{C}_{12} \mathrm{Ch}$ 的 $\pi$ 差别不大, 二者弹性模量的差别主要 是由第二方面原因造成. 对于 $\mathrm{C}_{12} \mathrm{Ch}$ 和 Ch-SDS (两 者每个高分子离子中含有等量的烷基基团), 尽管 $\mathrm{C}_{12} \mathrm{Ch}$ 的表面压低于 Ch-SDS, 但是弹性模量几乎相
同, 这反映了以共价键结合到 $\mathrm{C}_{12} \mathrm{Ch}$ 大分子上的烷 基链横向疏水相互作用导致凝胶结构形成, 因此对 弹性数值起到了明显作用. $\mathrm{C}_{12} \mathrm{Ch}$ 和 Ch-SDS 的界面 扩张粘性最大数值分别为 5 和 $20 \mathrm{mN} \cdot \mathrm{m}^{-1}$, 这种差 别主要是由于吸附层内烷基链横向疏水作用的强度 不同. $\mathrm{C}_{12} \mathrm{Ch}$ 烷基链疏水间相互作用的能量较高, 这 使得吸附层抵抗破裂的能力较强, 因此损失能量较 小, 粘性模量较小; Ch-SDS 复合物中, SDS 的烷基 链以相对较弱的静电作用结合到带相反电荷的大分 子链上, 虽然烷基链间也存在疏水相互作用, 然而振 荡变形时会导致其出现可逆的破裂变化, 这种周期 性的破裂和重建导致较大的能量损失, 因此粘性模 量较大.

我们研究了羟丙基甲基纤维素(HPMC)和具有 不同链长的季铵盐表面活性剂 $\mathrm{C}_{n} \mathrm{TAB}$ 混合溶液在 空气/水界面上的扩张粘弹性质 ${ }^{[5]}$. 发现 HPMC 加人 到不同浓度的 CTAB 溶液后, 体系的扩张弹性仍然 保持随频率增加不断上升的趋势, 但粘性部分则相 反, 而且溶液的扩张粘弹性对频率的依赖性大大降 低, 混合溶液的扩张弹性数值和单纯的 $\mathrm{C}_{n} \mathrm{TAB}$ 溶液 相比均明显降低. 图 2 示出了 HPMC/CTAB 混合溶 液的扩张弹性与单纯的 $\mathrm{CTAB}$ 溶液的比较结果, 图 中的理论计算值为 $\mathrm{CTAB}$ 溶液的扩张弹性数值与 单纯 $0.1 \mathrm{~g} \cdot \mathrm{L}^{-1} \mathrm{HPMC}$ 溶液的数值的简单加和. 显然, 实验测定结果远远低于理论计算值, 表明 HPMC 和 $\mathrm{CTAB}$ 在表面上存在相互作用和竞争吸附. HPMC 溶液中加人 $\mathrm{CTAB}$ 后, 体系的扩张弹性也显著低于 单纯的HPMC体系 $\left(0.05 \mathrm{~Hz}\right.$ 时, $0.1 \mathrm{~g} \cdot \mathrm{L}^{-1} \mathrm{HPMC}$ 的扩 张弹性数值为 $25.40 \mathrm{mN} \cdot \mathrm{m}^{-1}$ ), 此值高于 CTAB 的,

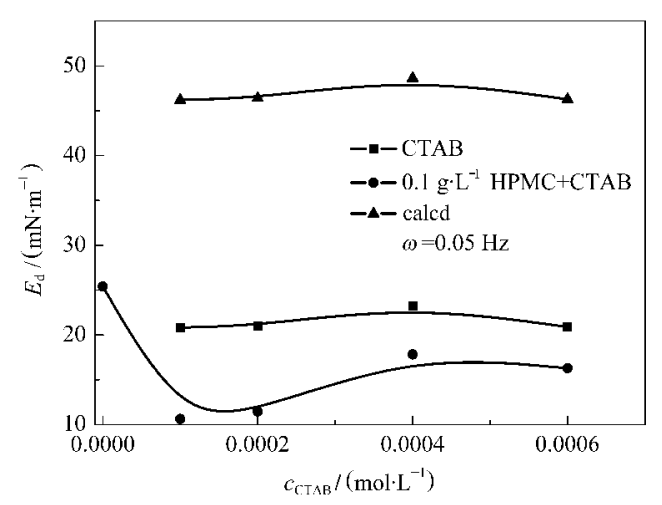

图 2 不同体系扩张弹性随 CTAB 浓度的变化 ${ }^{[55]}$

Fig.2 Dependence of dilational elasticity on CTAB concentration $^{[55]}$

CTAB: cetyltrimethylammonium bromide; HPMC: hydroxypropyl methyl cellulose 
表明该浓度范围内 HPMC 形成的界面膜的弹性和 强度与 $\mathrm{CTAB}$ 相比更高. 加人 $\mathrm{CTAB}$ 后由于 $\mathrm{CTAB}$ 具有较高的表面活性, 从体相向表面的扩散会将一 部分的 HPMC 分子从表面上替代下来, 因此导致扩 张弹性降低, 气/液界面膜的弹性和强度均下降.

比较疏水基长度影响时发现, $\mathrm{HPMC} /$ 十四烷基 三甲基溴化铵(TTAB)溶液的扩张弹性最高, HPMC/ CTAB 体系次之, HPMC/DTAB 的最低. DTAB 的扩 张弹性低于 $\mathrm{CTAB}$, 这主要是由于 DTAB 具有较短 的疏水链, 相对较弱的疏水作用导致其与 HPMC 形 成复合物的能力较弱, 因此界面上形成的膜相对于 $\mathrm{CTAB}$ 来说不够致密. 通过测定混合体系的表面张 力等温线对以上现象进行了解释: $\mathrm{HPMC} / \mathrm{C}_{n} \mathrm{TAB}$ 溶 液的表面张力变化分为三个阶段, 第一阶段为表面 上 HPMC 和表面活性剂分子共同存在; 第二阶段溶 液中增加的表面活性剂分子主要在体相中与 HPMC 形成复合物; 第三阶段溶液中开始形成正常 的表面活性剂胶束. 对三种表面活性剂界面粘弹性 的测定中, 所研究的 CTAB $\left(1 \times 10^{-4}-6 \times 10^{-4} \mathrm{~mol} \cdot \mathrm{L}^{-1}\right)$ 和 DTAB $\left(4 \times 10^{-4}-1.1 \times 10^{-2} \mathrm{~mol} \cdot \mathrm{L}^{-1}\right)$ 的浓度范围均处 在表面张力等温线的第二阶段, 此时表面上大部分 被表面活性剂分子所占据, 溶液中继续增加的 CTAB 或 DTAB 主要参与了复合物的形成. 而 TTAB 浓度范围 $\left(3 \times 10^{-5}-2 \times 10^{-3} \mathrm{~mol} \cdot \mathrm{L}^{-1}\right)$ 处在表面张 力曲线的第一阶段, 表面上有较多的 HPMC, 故混 合溶液的扩张弹性较高.

\section{2 合成大分子/表面活性剂混合体系}

尽管合成大分子/表面活性剂混合体系在实际 应用中起着重要的作用, 但是多年来则是集中在对 体相性质的研究 ${ }^{[56,57]}$. 近年来人们开始关注大分子/ 表面活性剂混合体系的界面流变行为, 这种方法可 以提供有关界面上结构形成和分子间相互作用本质 的重要信息 ${ }^{[58]}$.

\subsection{1 水溶性聚合物/表面活性剂混合体系}

聚乙烯吡咯烷酮 $(\mathrm{PVP})$ 和聚醚类大分子均为典 型的非离子水溶性聚合物, 已广泛应用于许多工业 领域. 我们通过界面流变性测定研究了这类大分子 与羧酸盐型阴离子表面活性剂的相互作用 ${ }^{[59]}$. PVP/ 油酸钠 $\left(\mathrm{C}_{17} \mathrm{H}_{33} \mathrm{COONa}\right)$ 混合体系的界面扩张粘弹性 结果表明, 混合体系的 $E$ 最大值所对应的浓度(12 $\mathrm{mg} \cdot \mathrm{L}^{-1}$ ) 低于单纯的 $\mathrm{C}_{17} \mathrm{H}_{33} \mathrm{COONa}$ 所对应的油酸钠 浓度 $\left(120 \mathrm{mg} \cdot \mathrm{L}^{-1}\right)$, 这表明PVP的加人极大地增强了 体系的界面粘弹性. 当混合体系中 $\mathrm{C}_{17} \mathrm{H}_{33} \mathrm{COONa}$ 浓
度超过 $400 \mathrm{mg} \cdot \mathrm{L}^{-1}$ ( $\approx \mathrm{cac}, \mathrm{cac}$ 为临界聚集浓度)时, 界面上 $\mathrm{C}_{17} \mathrm{H}_{33} \mathrm{COONa} / \mathrm{PVP}$ 膜的 $E$ 值降低, 几乎接近 于纯 $\mathrm{C}_{17} \mathrm{H}_{33} \mathrm{COONa}$ 的模量值. 由此可以推断, 对于 纯 PVP 溶液而言, PVP 分子吸附在界面上, 它们之 间形成分子内或分子间聚集体，当向其中加人 $\mathrm{C}_{17} \mathrm{H}_{33} \mathrm{COONa}$ 后, 相互作用形成了 $\mathrm{C}_{17} \mathrm{H}_{33} \mathrm{COONa} /$ PVP 复合物. 随着 $\mathrm{C}_{17} \mathrm{H}_{33} \mathrm{COONa}$ 浓度增加, 更多的 $\mathrm{C}_{17} \mathrm{H}_{33} \mathrm{COONa}$ 分子吸附在界面上, PVP 和 $\mathrm{C}_{17} \mathrm{H}_{33} \mathrm{COONa}$ 分子之间的疏水相互作用使得几乎所 有的 PVP 分子从界面转移到体相中, 这样溶液界面 上几乎被 $\mathrm{C}_{17} \mathrm{H}_{33} \mathrm{COONa}$ 分子所占据, 因此最终混合体 系的表面张力和 $E$ 值都几乎与纯 $\mathrm{C}_{17} \mathrm{H}_{33} \mathrm{COONa}$ 体系 相同. 当溶液中存在 $\mathrm{NaCl}$ 和 $\mathrm{NaI}$ 时, $\mathrm{C}_{17} \mathrm{H}_{33} \mathrm{COONa} / \mathrm{PVP}$ 溶液的最大 $E$ 值出现在 $2.5 \mathrm{mg} \cdot \mathrm{L}^{-1}$, 这是因为 $\mathrm{NaX}$ 的 加人使 $\mathrm{C}_{17} \mathrm{H}_{33} \mathrm{COONa} / \mathrm{PVP}$ 复合物的形成出现在更低 的浓度. 而且 $\mathrm{NaCl}$ 在较低浓度时就可使 $E$ 值迅速降 低, 此现象可用盐促进了PVP和 $\mathrm{C}_{17} \mathrm{H}_{33} \mathrm{COONa}$ 之间 的相互缔合来解释.

L64 $\left(\mathrm{PEO}_{13} \mathrm{PPO}_{30} \mathrm{PEO}_{13}\right)$ 是 Pluronic 嵌段聚醚的 一种, 摩尔比为 $1: 1 、 1: 3$ 和 1:5 的 $\mathrm{L} 64 / \mathrm{C}_{17} \mathrm{H}_{33} \mathrm{COONa}$ 复配体系 $E$ 值与扩张频率和体系的组成均有关. 随 着 $\mathrm{C}_{17} \mathrm{H}_{33} \mathrm{COONa}$ 在混合体系中摩尔比的增加, 扩张 模量的最大值出现在更低的 L64 浓度(如图 3) ${ }^{[60]}$. 例 如, 在扩张频率为 $0.1 \mathrm{~Hz}, \mathrm{~L} 64$ 与 $\mathrm{C}_{17} \mathrm{H}_{33} \mathrm{COONa}$ 的摩 尔比为 $1: 1$ 和 $1: 3$ 时, $\mathrm{L} 64 / \mathrm{C}_{17} \mathrm{H}_{33} \mathrm{COONa}$ 复配体系扩 张模量的最大值分别出现在 1 和 $0.1 \mathrm{mg} \cdot \mathrm{L}^{-1}$, 而 $1: 5$ 的 $\mathrm{L} 64 / \mathrm{C}_{17} \mathrm{H}_{33} \mathrm{COONa}$ 复配体系的扩张模量最大值分 别出现在 $0.01 \mathrm{mg} \cdot \mathrm{L}^{-1}$. 这表明 $\mathrm{C}_{17} \mathrm{H}_{33} \mathrm{COONa}$ 加人到 L64 溶液中提高了体系的表面活性.

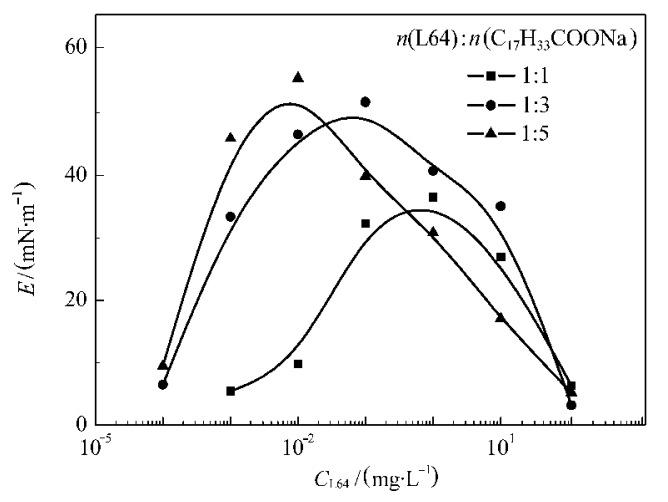

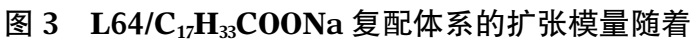
L64 浓度的变化 ${ }^{[60]}$

Fig.3 Variation of dilational modulus as a function of $\mathrm{L} 64$ concentration for $\mathrm{L} 64 / \mathrm{C}_{17} \mathrm{H}_{33} \mathrm{COONa}$ mixed system ${ }^{[60]}$ L64: $\mathrm{PEO}_{13} \mathrm{PPO}_{30} \mathrm{PEO}_{13} ; \omega=0.1 \mathrm{~Hz}$ 
在 CTAB 浓度为 $1.0 \times 10^{-4} \mathrm{~mol} \cdot \mathrm{L}^{-1}$ 时达到最大值,

$\mathrm{CTAB}$ 浓度高于其 $\mathrm{cmc}$ 后, 混合体系的 $E$ 值与单独 表面活性剂溶液的数值接近, 且该数值很小, L64/ $\mathrm{CTAB}$ 体系的粘性部分随频率增加也基本不变. 与 L64 具有相近分子量的 $17 \mathrm{R} 4\left(\mathrm{PPO}_{14} \mathrm{PEO}_{24} \mathrm{PPO}_{14}\right) /$ $\mathrm{CTAB}$ 混合体系的 $E$ 值远低于 $17 \mathrm{R} 4$ 和 CTAB 单独 溶液模量值的简单加和, 甚至低于单纯 CTAB 的模 量值, 而且随着 CTAB 浓度增加, $E$ 值及其粘性部分 均在 $c_{\mathrm{CTAB}}=3 \times 10^{-4} \mathrm{~mol} \cdot \mathrm{L}^{-1}$ 出现一个最大值, 而单独 $\mathrm{CTAB}$ 在浓度为 $3 \times 10^{-6} \mathrm{~mol} \cdot \mathrm{L}^{-1}$ 时出现最大值. 这说 明 17R4 与 CTAB 在界面发生了相互作用. 但当 CTAB 浓度达到 $1 \times 10^{-3} \mathrm{~mol} \cdot \mathrm{L}^{-1}$ 时, 混合溶液的 $E$ 值及其粘性部分之值与纯 CTAB 者比较接近, 说明 此时界面膜上的 17R4 分子完全被小分子活性剂 CTAB 取代. Pluronic 嵌段共聚物与小分子表面活 性剂的作用机理主要是共聚物的 PPO 段与活性剂 的烷烃链间的疏水作用. 可以推断, 随着 CTAB 浓 度的增加, CTAB 疏水基团与 PPO 段发生相互作用, 界面上形成 17R4/CTAB 的复合物, 这导致 17R4 的 疏水性减弱, 亲水性增强, 混合膜的 $E$ 值有增大的 趋势, 继续增大 CTAB 浓度致使界面上的 17R4 分 子被完全结合, 复合物进入体相溶液, 界面完全被 CTAB 分子所取代, 此时混合膜的扩张粘弹性表现 为单独 $\mathrm{CTAB}$ 的粘弹性. $E$ 达到最大值; 17R4/ $\mathrm{CTAB}$ 混合体系的 $E$ 值及其粘性部分均大于 L64/ CTAB 体系的, 说明 17R4/CTAB 混合界面膜的强度 高于后者; 且混合膜的粘弹性数值与 $17 \mathrm{R} 4$ 和 L64 单独体系模量值的相对大小关系一致, 这说明随着 界面复合物的形成, 混合体系扩张粘弹性仍主要由 共聚物决定 ${ }^{[6]]}$.

聚电解质是一类应用非常广泛的荷电大分子. 荷相反电性的聚电解质/表面活性剂混合体系界面 流变性质的研究也备受重视 ${ }^{[62]}$. 其一般性规律是, 表 面活性剂浓度较低时, 吸附层通常呈现粘弹性行为, 这是由于聚电解质/表面活性剂两组分在界面上的 协同吸附; 当表面活性剂浓度较高时, 由于聚电解 质从界面上发生解吸, 表面几乎被表面活性剂分子 占据, 此时为流体界面 ${ }^{[4]}$. Miller 等 ${ }^{\left[{ }^{[3]}\right.}$ 报道了聚苯乙 烯磺酸钠(PSS)/DTAB 混合体系在气/液界面上随时 间和表面活性剂浓度变化的扩张流变行为. 可以将 研究的整个表面活性剂浓度范围划分为两个具有不 同界面粘弹性质的区域, 而在这之间存在一个界面
粘弹性发生突变的窄小区域. 当表面活性剂浓度较 低时, 扩张粘弹性数值随着时间的延长逐渐增大至 60-100 $\mathrm{mN} \cdot \mathrm{m}^{-1}$, 表明表面层内 PSS 和 DTAB 发生 强烈的相互作用. 而且此时 PSS 的界面流变行为与 高浓度的 PSS 溶液是一致的, 用经典 Goddard 模型 不能解释此区域内的实验结果, 因此 Miller 等 ${ }^{\left[{ }^{[3]}\right]}$ 对 该模型进行了修正, 他们考虑了聚苯乙烯链的疏水 本质, 认为表面活性剂碳氢链与聚苯乙烯主链链段 之间, 链段以及碳氢链之间的相互作用形成二维聚 集体结构, 由于聚电解质不同链问互相纠缠和疏水 基团之间的结合使得整个吸附层呈现刚性, 因此 $E$ 值很高. 窄小区域的 $E$ 值突然降低则是由于在表面 层的二维聚集体结构转化为微凝胶结构, 这一假设 与椭圆光度法所测结果一致. 表面活性剂浓度继续 增大导致表面层内结构松散, 因此 $E$ 值下降. 结果 也说明界面粘弹性对聚合物构象的变化是比较敏感 的. Noskov 等 ${ }^{[6] 1}$ 研究了聚二甲基丙基二甲基氯苯胺 (PDAC)/SDS 混合体系的界面扩张流变行为, 得到 了与 PSS 和 DTAB 吸附膜类似的结果. 表面活性剂 浓度较低时, 聚合物/表面活性剂复合物具有很高的 表面活性, 因此“最接近区域”PDAC 浓度很高, 较低 的表面活性剂浓度不能完全中和 PDAC 链段的电 荷, 结果刚性吸附层内, 由于荷电基团的排斥力使得 $E$ 值较高. 当表面活性剂的浓度达到带电荷的聚合 物单体浓度时, 界面上微粒的形成导致 $E$ 值下降. 但通过以上这两个体系所得到的表面活性剂浓度对 扩张模量的依赖性关系并不是所有聚电解质/表面 活性剂的特征, 而与电解质分子结构密切相关.

油/水界面与气/液表面不同. 在油/水界面上, 油

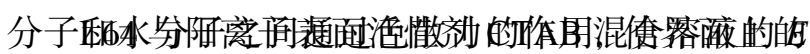
油分子和水分子受到了异相分子吸引, 有可能形成 一种“两亲性界面缔合物”. 江龙院士等 ${ }^{[65]}$ 研究表面 活性剂/聚丙烯酰胺在油/水界面的流变性时证实了 这一点. 我们 ${ }^{[66}$ 也研究了含有不同浓度 $\operatorname{HPAM}($ 部分 水解聚丙烯酰胺)时 CTAB水溶液/液体石蜡的界面 粘弹性. 固定 $\mathrm{CTAB}$ 浓度为 $1.0 \times 10^{-4} \mathrm{~mol} \cdot \mathrm{L}^{-1}, \mathrm{HPAM}$ 的浓度分别为 $10 、 20$ 和 $50 \mathrm{mg} \cdot \mathrm{L}^{-1}$, 体系的界面粘弹 性如图 4 所示. 可以看出, 随着 HPAM 含量增加, $E$ 值逐渐增大; 而相角 $\tan \theta$ 降幅很大, 这说明弹性模 量对扩张模量的贡献增大, 或者界面的弹性增强. 可 能的原因是, 当 CTAB/HPAM 混合界面层发生扩张 或压缩时, 界面吸附分子会产生两种弛豫行为, 一种 是 CTAB 分子在体相和界面之间的扩散交换过程, 

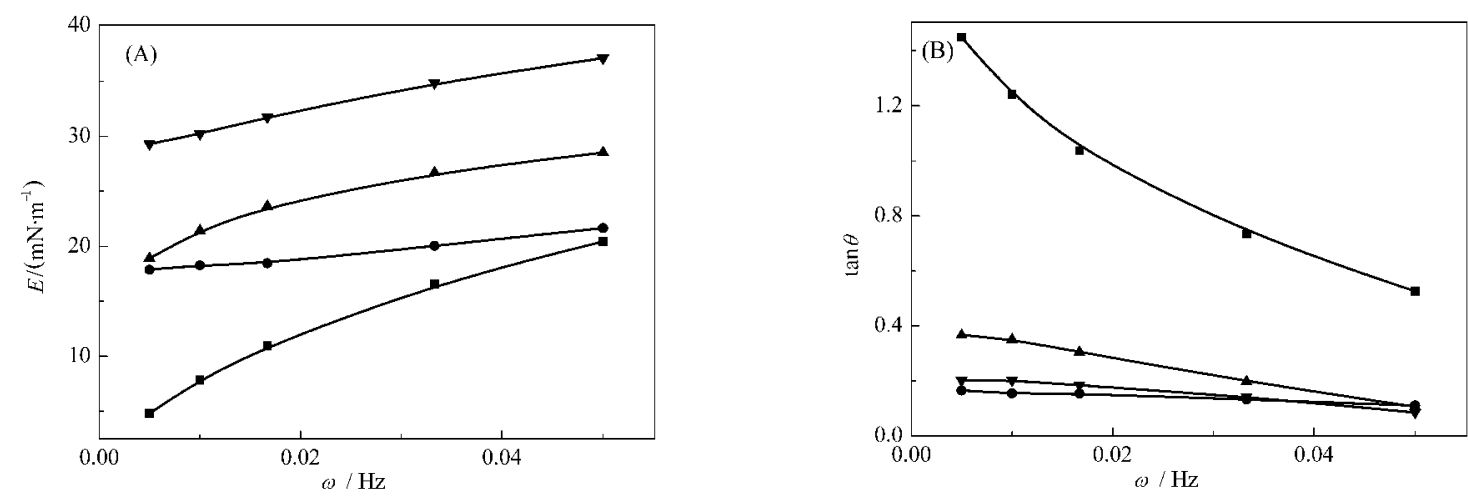

图 4 不同 HPAM 浓度时 CTAB 的油/水界面粘弹性随扩张频率的变化 ${ }^{[66]}$

Fig.4 Dependence of oil/water interfacial dilational viscoelasticity of CTAB on oscillating frequency at different HPAM concentrations ${ }^{[6]}$

(A) dilational modulus, (B) $\tan \theta ; c(\mathrm{HPAM}) /\left(\mathrm{mg} \cdot \mathrm{L}^{-1}\right):($

) 50 ; $c$ (CTAP): $1.0 \times 10^{-4} \mathrm{~mol} \cdot \mathrm{L}^{-1}$; HPAM: hydrolyzed polyacrylamide

另外一种是界面上 CTAB/HPAM 复合物弛豫行为, 例如大分子链的卷曲或伸展、分子取向等过程, 与单 独 CTAB 的情况相比, 当界面层面积发生相同变化 时, 混合体系的界面张力变化更多, 相应的 $E$ 值也 更大.

\subsection{2 疏水修饰的聚合物/表面活性剂混合体系}

疏水缔合水溶性聚合物(HAWSP) 是近年来备 受关注的一种新材料, 在一定浓度时疏水基团之间 相互结合因而溶液粘度明显增加. 此外, 这类聚合物 还具有抗盐、抗剪切等特殊性质. 尤其是由于丙烯酰 胺类水溶性非离子聚合物常作为粘度调节剂用在三 次采油中而引起了人们的特别关注 ${ }^{[67]}$. 近年来, 国内 外对疏水缔合共聚物/表面活性剂混合体系的表面 张力、粘度等体相流变性质研究较多, 但对其界面流 变性质的研究则较为少见. Zhao 课题组 ${ }^{[68,609}$ 在这方 面做了大量工作.

赵濉等 ${ }^{[36}$ 采用界面张力弛豫法研究了疏水缔合 聚合物聚丙烯酰胺/2-乙基己基丙烯酸酯(P(AM/2EHA))在正辛烷/水界面上的扩张粘弹性质, 考察了 不同类型表面活性剂 SDS、辛基酚聚氧乙烯醚(TX100) 和 CTAB 对其界面扩张性质的影响. 研究发 现, 界面上的表面活性剂分子可以与聚合物的疏水 嵌段形成类似混合胶束的聚集体,表面活性剂分子 与聚集体之间存在快速交换. 这种驰豫过程的特征 时间远比分子在体相与界面间的扩散交换时间短. 当界面面积增大时, 上述混合胶束中的表面活性剂 分子能快速释放, 在界面层内原位快速消除界面张 力梯度, 从而大大降低界面扩张弹性. 界面上的 $\mathrm{CTAB}$ 分子与聚合物链节上的负电中心通过较强的
电荷吸引作用形成复合物. 当界面面积增大时, 上述 混合胶束中的 CTAB 分子释放较慢, 界面张力梯度 较大. 非离子表面活性剂 TX-100 分子量较大, 扩散 速率较慢, 它在界面上与聚集体间的交换比阴离子 表面活性剂 SDS 慢, 其特征时间约为 $0.9 \mathrm{~s}$.

Wang 等 ${ }^{[70]}$ 还通过界面张力弛豫法研究了疏水 缔合聚合物聚丙烯酰胺/2-苯乙基丙烯酸( $\mathrm{P}(\mathrm{AM} /$ POEA))和 SDS 混合体系在辛烷/水界面的扩张粘弹 性质. 当聚合物溶液浓度为 $5000 \mathrm{mg} \cdot \mathrm{L}^{-1}$ 时, 随着 SDS 的加人, 极限扩张弹性数值不断减小, 粘性部 分随振荡频率的增加出现最大值, 而且 SDS 浓度越 大, 出现最大值所对应的频率越小. 这是因为界面上 的大分子被 SDS 取代, 分子间相互作用减弱. 当聚 合物溶液浓度为 $200 \mathrm{mg} \cdot \mathrm{L}^{-1}$ 时, 随 SDS 的加人, 极 限扩张弹性数值先增大后减小, 这可能是因为界面 上聚合物浓度较低, 即使在很低的表面活性剂浓度 下, 吸附在界面上的 SDS 分子也决定了界面膜的扩 张粘弹性质. 继续增大 SDS 浓度, 界面上的大分子 逐渐被 SDS 取代, 导致极限扩张弹性数值降低.

\section{4 结束语}

以上综述表明, 扩张流变技术已成为研究大分 子与表面活性剂相互作用的简便而有效的实验手 段. 通过分析界面扩张粘弹性数据和界面弛豫过程 的特征参数, 可以得知分子间的相互作用以及分子 在界面上可能存在的聚集状态等信息, 阐明界面膜 的微观性质, 这对实际生产和应用具有重要的理论 指导意义. 今后, 随着界面扩张流变学以及其它技术 的进一步发展和综合应用, 如果既能获得吸附层的 
变化, 又可给出吸附层结构和组成的可靠的定量信 息, 并能有效地确定泡沫和乳状液的稳定性与界面 扩张流变性之间的对应关系, 那么, 含有大分子/表 面活性剂混合体系对于工业生产和生命过程等必将 发挥越来越重要的作用.

\section{References}

1 Lucassen-Reynders, E. H. Physical chemistry of surfactant action (Surfactant Science Series Vol. 11). New York: Marcel Dekker Inc., 1981: 131

2 Goddard, E. D.; Ananthanthapadmanabhan, K. P. Interactions of surfactants with polymers and proteins. New York: CRC Press, Inc., 1993: 1

3 Kwak, J. C. T. Polymer-surfactant systems. New York: Marcel Dekker Inc., 1998: 267

4 Taylor, D. J. F.; Thomas, R. K.; Penfold, J. Adv. Colloid Interface Sci., 2007, 132: 69

5 Xu, G. Y.; Luan, Y. X.; Liu, J.; Yu, L. Acta Phys. -Chim. Sin., 2005, 21(5): 577 [徐桂英, 奕玉霞, 刘 静, 于 丽. 物理化学 学报, 2005, 21(5): 577]

6 Wu, D.; Xu, G. Y. Acta Phys. -Chim. Sin., 2006, 22(2): 254 [吴 丹, 徐桂英. 物理化学学报, 2006, 22(2): 254]

7 Luan, Y. X.; Xu, G. Y.; Chen, A. M. Acta Phys. -Chim. Sin., 2003, 19(2): 185 [栾玉霞, 徐桂英, 陈爱民. 物理化学学报, 2003, 19 (2): 185]

8 Li, Y. M.; Yuan, S. L.; Xu, G. Y. Acta Phys. -Chim. Sin., 2003, 19 (10): 986 [李一鸣, 苑世领, 徐桂英. 物理化学学报, 2003, 19 (10): 986]

9 Regismond, S. T. A.; Gracie, K. D.; Winnik, F. M.; Goddard, E. D. Langmuir, 1997, 13: 5558

10 Chari, K.; Seo, Y. S.; Satija, S. J. Phys. Chem. B, 2004, 108: 11442

11 Monteux, C.; Williams, C. E.; Meunier, J.; Anthony, O.; Bergeron, V. Langmuir, 2004, 20: 57

12 Lynch, I.; Piculell, L. J. Phys. Chem. B, 2004, 108: 7515

13 Chari, K.; Antalek, B.; Kowalczyk, J.; Eachus, R. S.; Chen, T. J. Phys. Chem. B, 1999, 103: 9867

14 He, Q.; Zhang, Y.; Lu, G.; Miller, R.; Möhwald, H.; Li, J. Adv. Colloid Interface Sci., 2008, 140: 67

15 Richardson, R. M.; Pelton, R.; Cosgrove, T.; Zhang, J. Macromolecules, 2000, 33: 6269

16 Noskov, B. A.; Alexandrov, D. A.; Loglio, G.; Miller, R. Colloids Surf. A, 1999, 156: 307

17 Patino, J. M. R.; Fernández, M. C.; Sánchez, C. C.; Niño, M. R. R. J. Colloid Interface Sci., 2007, 313: 141

18 Blomqvist, B. R.; Warnheim, T.; Claesson, P. M. Langmuir, 2005, 21: 6373

19 Monteux, C.; Mangeret, R.; Laibe, G.; Freyssingeas, E.; Bergeron, V.; Fuller, G. Macromolecules, 2006, 39: 3408

20 Murray, B. S. Curr. Opin. Colloid Interface Sci., 2002, 7: 426
21 Bonfillon, A.; Langevin, D. Langmuir, 1993, 9: 2172

22 Monteux, C.; Fuller, G. G.; Bergeron, V. J. Phys. Chem. B, 2004, 108: 16473

23 Li, T. M.; Zhao, L. Y. Acta Phys. -Chim. Sin., 1996, 12(8):709 [李佟茗, 赵丽燕. 物理化学学报, 1996, 12(8): 709]

24 Monroy, F.; Kahn, J. G.; Langevin, D. Colloids Surf. A, 1998, 143: 251

25 Kovalchuk, V. I.; Krägel, J.; Makievski, A. V.; Ravera, F.; Liggieri, L.; Loglio, G.; Fainerman, V. B.; Miller, R. J. Colloid Interface Sci., 2004, 280: 498

26 Ravera, F.; Ferrari, M.; Santini, E.; Liggieri, L. Adv. Colloid Interface Sci., 2005, 117: 75

27 Kovalchuk, V. I.; Miller, R.; Fainerman, V. B.; Loglio, G. Adv. Colloid Interface Sci., 2005, 114: 303

28 Stubenrauch, C.; Fainerman, V. B.; Aksenenko, E. V.; Miller, R. J. Phys. Chem. B, 2005, 109: 1505

29 Erni, P.; Fischer, P.; Windhab, E. J. Langmuir, 2005, 21: 10555

30 Kausch, C. M.; Kim, Y.; Russell, V. M.; Medsker, R. E.; Thomas, R. R. Langmuir, 2003, 19: 7354

31 Bai, Y.; Xu, G. Y.; Xin, X.; Sun, H. Y.; Zhang, H. X.; Hao, A. Y.; Yang, X. D.; Yao, L. Colloid Polym. Sci., 2008, 286: 1475

32 Hansen, F. K. Langmuir, 2008, 24: 189

33 Li, J. B.; Kretzschmar, G.; Miller, R.; Möhwald, H. Colloids Surf. A, 1999, 149: 491

34 Zhang, C. R.; Li, Z. Q.; Luo, L.; Zhang, L.; Song, X. W.; Cao, X. L.; Zhao, S.; Yu, J. Y. Acta Phys. -Chim. Sin., 2007, 23(2): 247 [张春荣, 李振泉, 罗 澜, 张 路, 宋新旺, 曹绪龙, 赵 濉, 俞稼 镛. 物理化学学报, 2007, 23(2): 247]

35 Tadros, T. F. Colloid stability: The role of surface forces-Part I. Weinheim: Wiley-Vch., 2007: 295

36 Wang, D. X.; Luo, L.; Zhang, L.; Wang, Y. Y.; Zhao, S.; Yu, J. Y. Acta Phys. -Chim. Sin., 2005, 21(11): 1205 [王东贤, 罗 澜, 张 路, 王宜阳, 赵 濉, 俞稼镛. 物理化学学报, 2005, 21(11): 1205]

37 Noskov, B. A.; Akentiev, A. V.; Bilibin, A. Y.; Zorin, I. M.; Miller, R. Adv. Colloid Interface Sci., 2003, 104: 245

38 Noskov, B. A.; Akentiev, A. V.; Loglio, G.; Miller, R. J. Phys. Chem. B, 2000, 104: 7923

39 Noskov, B. A.; Akentiev, A. V.; Miller, R. J. Colloid Interface Sci., 2002, 255: 417

40 Pereira, L. G. C.; Théodoly, O.; Blanch, H. W.; Radke, C. J. Langmuir, 2003, 19: 2349

41 Girardet, J. M.; Humbert G.; Creusot, N.; Chardot, V.; Campagna, S.; Courthaudon, J. L.; Gaillard, J. L. J. Colloid Interface Sci., 2001, 243: 515

42 Miller, R.; Fainerman, V. B.; Makievski, A. V.; Kragela, J.; Grigorieva, D. O.; Kazakov, V. N.; Sinyachenko, O. V. Adv. Colloid Interface Sci., 2000, 86: 39

43 Murray, B. S.; Ventura, A.; Lallemant, C. Colloids Surf. A, 1998, 143: 211

44 Patino, J. M. R.; Fernández, M. C.; Niño, M. R. R.; Sánchez, C. C. 
Biomacromolecules, 2006, 7: 2661

45 Blomqvist, B. R.; Ridout, M. J.; Mackie, A. R.; Wárnheim, T.; Claesson, P. M.; Wilde, P. Langmuir, 2004, 20: 10150

46 Aksenenko, E. V.; Kovalchuk, V. I.; Fainerman, V. B.; Miller, R. Adv. Colloid Interface Sci., 2006, 122: 57

47 Rao, A.; Kim, J.; Thomas, R. R. Langmuir, 2005, 21: 617

48 Cooke, D. J.; Dong, C. C.; Thomas, R. K.; Howe, A. M.; Simister, E. A.; Penfold, J. Langmuir, 2000, 16: 6546

49 Wu, D.; Xu, G. Y.; Feng, Y. J.; Li, Y. M. Int. J. Biol. Macromol., 2007, 40: 345

50 Latnikova, A. V.; Lin, S. Y.; Loglio, G.; Miller, R.; Noskov, B. A. J. Phys. Chem. C, 2008, 112: 6126

51 Kurita, K.; Akao, H.; Yang, J.; Shimojoh, M. Biomacromolecules, 2003, 4: 1868

52 Grant, J.; Cho, J.; Allen, C. Langmuir, 2007, 23: 4688

53 Keda, S.; Kumagai, H. J. Agric. Food Chem., 1997; 45: 3452

54 Babak, V. G.; Desbrières, J. Mendeleev Commun., 2005, 15: 35

55 Li, Y. M.; Xu, G. Y.; Xin, X.; Cao, X. R.; Wu, D. Carbohydr. Polym., 2008, 72: 211

56 Kogej, K.; Skerjanc, J. Langmuir, 1999, 15: 4251

57 Kogej, K.; Theunissen, E.; Reynaers, H. Langmuir, 2002, 18: 8799

58 Zhang, J. Y.; Wang, X. P.; Liu, H. Y.; Tang, J. A.; Jiang, L. Colliods Surf. A, 1998, 132: 9

59 Xin, X.; Xu, G. Y.; Wu, D.; Gong, H. J.; Zhang, H. X.; Wang, Y. J. Colliods Surf. A, 2008, 322: 54
60 Xin, X.; Xu, G. Y.; Wang, Y. J.; Mao, H. Z.; Zhang, Z. Q. Eur. Polym. J., 2008, in press

61 Shi, X. F. Master Dissertation. Jinan: Shandong University, 2008 [史晓峰. 硕士学位论文. 济南: 山东大学, 2008]

62 Ritacco, H.; Kurlat, D.; Langevin, D. J. Phys. Chem. B, 2003, 107: 9146

63 Noskov, B. A.; Loglio, G.; Miller, R. J. Phys. Chem. B, 2004, 108 18615

64 Noskov, B. A.; Grigoriev, D. O.; Lin, S. Y.; Loglio, G.; Miller, R. Langmuir, 2007, 23: 9641

65 Wang, X. P.; Zhang, J. Y.; Tang, J. A.; Jiang, L. Acta Phys. -Chim. Sin., 1998, 14(1): 88 [王新平, 张嘉云, 唐季安, 江 龙. 物理化 学学报, 1998, 14(1): 88]

66 Zhang, H. X.; Xu, G. Y.; Wu, D.; Wang, S. W. Colliods Surf. A, 2008, 317: 289

67 Wang, Y.Y.; Dai, Y. H.; Zhang, L.; Luo, L.; Chu, Y. P.; Zhao, S.; Li, M. Z.; Wang, E. J.; Yu, J. Y. Macromolecules, 2004, 37: 2930

68 Sun, T. L.; Zhang, L.; Wang, Y. Y.; Zhao, S.; Peng, B.; Li, M. Y.; Yu, J. Y. J. Colloid Interface Sci., 2002, 255: 241

69 Wang, Y. Y.; Zhang, L.; Sun, T. L.; Zhao, S.; Yu, J. Y. J. Colloid Interface Sci., 2003, 270: 163

70 Wang, Y. Y.; Dai, Y. H.; Zhang, L.; Tang, K.; Luo, L.; Gong, Q. T. Zhao, S.; Li, M. Z.; Wang, E. J.; Yu, J. Y. J. Colloid Interface Sci., 2004, 280: 76 